\title{
Preterm feeding recommendations are achievable in large-scale research studies
}

Leila Cheikh Ismail 1,12*, Francesca Giuliani ${ }^{2}$, Bashir A. Bhat ${ }^{3}$, Deborah Bishop', Aris T. Papageorghiou1', Roseline Ochieng ${ }^{4}$, Fabien Puglia', Douglas G. Altman ${ }^{5}$, Michael Maia-Schlüssel $^{1}$, Julia A. Noble ${ }^{6}$, Enrico Bertino ${ }^{2}$, Michael G. Gravett ${ }^{7}$, Manorama Purwar ${ }^{8}$, Lui Yajing ${ }^{9}$, Denise Mota ${ }^{10}$, Eric Ohuma ${ }^{1,5}$, Ann Lambert ${ }^{1}$, Stephen H. Kennedy ${ }^{1}$, Zulfiqar A. Bhutta ${ }^{11}$, José Villar $^{1}$ and for the International Fetal and Newborn Growth Consortium for the $21^{\text {st }}$ Century (INTERGROWTH-21 ${ }^{\text {st }}$ )

\begin{abstract}
Background: The INTERGROWTH-21 ${ }^{\text {st }}$ Project aimed to produce international, prescriptive, postnatal growth standards for preterm infants born to healthy, well-nourished mothers receiving adequate antenatal care. There is little information available regarding optimal postnatal growth among uncomplicated preterm newborns. We describe how the preterm infants contributing to the standards followed evidence-based feeding recommendations.

Methods: In the Fetal Growth Longitudinal Study (FGLS), a component of the INTERGROWTH-21 ${ }^{\text {st }}$ Project, fetal growth was monitored by ultrasound from $<14$ weeks' gestation until birth in pregnancies at low risk of adverse outcomes. All preterms $\left(\geq 26^{+0}\right.$ and $<37^{+0}$ weeks' gestation) were followed up during infancy. Internationallyaccepted feeding recommendations for preterms, agreed by the INTERGROWTH-21 ${ }^{\text {st }}$ Neonatal Group, were implemented at each study site. Standardised questionnaires served to record information on their feeding practices.
\end{abstract}

Results: Feeding data were collected from 201 eligible preterms. The median (interquartile range, IQR) gestational age at birth was 36.0 (35.0 - 36.6) weeks. The prevalence of any breastfeeding was $82 \%$ within $72 \mathrm{~h}$ of birth, $96 \%$ at 2 weeks, $82 \%$ at 4 months and $70 \%$ at 8 months postnatal age. The figures for exclusive breastfeeding were $51 \%$ within 72 h of birth, $72 \%$ at hospital discharge, $49 \%$ at 4 months, $38 \%$ at 5 months and $12 \%$ at 6 months. Complementary foods were introduced at a median (IQR) postnatal age of $6.0(5.1-6.8)$ months.

Conclusion: Most preterms were exclusively breastfed upon hospital discharge, and breastfeeding remained a substantial source of nutrition throughout the study. Recommendations, centred on breastfeeding, were adequately followed within the expected variation of such diverse settings.

Keywords: Preterm infants, Nutrition, Breastfeeding, Postnatal growth and development

\footnotetext{
* Correspondence: leila.cheikhismail@obs-gyn.ox.ac.uk

Stephen H. Kennedy, Zulfiqar A. Bhutta and José Villar are joint senior

authors.

${ }^{1}$ Nuffield Department of Obstetrics \& Gynaecology and Oxford Maternal \&

Perinatal Health Institute, Green Templeton College, University of Oxford,

Oxford, UK

${ }^{12}$ Nuffield Department of Obstetrics \& Gynaecology, Level 3 Women's Centre,

John Radcliffe Hospital, Oxford OX3 9DU, UK

Full list of author information is available at the end of the article
} 


\section{Background}

INTERGROWTH-21 ${ }^{\text {st }}$ is a multi-country project to develop international, prescriptive standards for fetal growth, newborn size and preterm postnatal growth [1], to complement the existing WHO Child Growth Standards [2, 3]. Such standards describe how individuals should grow, as opposed to reference charts that describe how individuals have grown at particular times and locations [4]. The aim of the Postnatal Preterm Followup Study (PPFS), a component of the INTERGROWTH$21^{\text {st }}$ Project was to construct prescriptive standards to monitor the postnatal growth of preterm infants following our a priori conceptual framework [4]. Using a similar approach to the WHO Multicentre Growth Reference Study (MGRS) [2, 3], we studied a cohort of uncomplicated, preterm infants born to healthy, well-nourished women from geographically diverse populations, who received adequate antenatal care and were at low risk of adverse maternal and perinatal outcomes, and who were not exposed to any known non-microbiological contamination or toxic substances [5].

Preterm infants are a heterogeneous group, varying by gestational age at birth and the severity of their comorbidities, many of which are associated with being born at a very early gestational age and growth restricted [6]. Some of the comorbidities are also probably associated with immature gastrointestinal function, resulting in an imbalance between rapid growth, nutrient supply and the adequacy of the metabolic response, occurring during the unplanned transition from trans-placental nutrition to oral feeding $[7,8]$.

There is controversy, both conceptually and at a practical level, about how best to feed preterms; hence, feeding practices vary among and within countries [9]. Some practices have been proposed, which should support a tissue accretion rate matching that of normal intrauterine growth. This approach assumes that the growth rate of a fetus of a comparable gestational age is desirable for the postnatal growth, development and long-term health of a preterm. However, such a pattern is seldom attained in preterm neonates, who almost always show a postnatal cumulative nutritional deficit and so-called 'extrauterine growth restriction' [10]. Feeding guidelines for term infants are based on the substantial benefits of breast milk, e.g. decreased rates of infection and mortality, and better long-term outcomes, including improved neurocognitive and cardiovascular development. There is increasing evidence that there are also important benefits to be gained from giving the mother's milk to sick and preterm infants in neonatal intensive care units [11]. However, their nutritional needs are different from those of term infants and should be evaluated carefully, especially bearing in mind the need to fortify human milk [11].
Therefore, in developing the preterm prescriptive growth standards, it was essential to ensure that the recommended feeding practices for optimal postnatal growth in preterms were implemented, including the provision of supplements and complementary foods when necessary. In this paper, we describe the breastfeeding and complementary feeding practices of the infants enrolled in PPFS.

\section{Methods}

Study design

INTERGROWTH-21 ${ }^{\text {st }}$ was a population-based project conducted between 2009 and 2014, in eight urban areas: the cities of Pelotas, Brazil; Turin, Italy; Muscat, Oman; Oxford, UK; Seattle, USA; Shunyi County, a suburban district of the Beijing municipality, China; the central area of the city of Nagpur (Central Nagpur), Maharashtra, India; and the Parklands suburb of Nairobi, Kenya [1].

All singleton, preterm newborns $\left(\geq 26^{+0}\right.$ to $<37^{+0}$ weeks' gestation) in the Fetal Growth Longitudinal Study (FGLS) component of INTERGROWTH-21 ${ }^{\text {st }}$, whose intrauterine growth was monitored by ultrasound, were eligible for PPFS. The detailed methodology has been published elsewhere [1]. Briefly, infants contributing to the standards must have: a) been free of major congenital malformations and severe neonatal conditions associated with impaired postnatal growth; b) received standardised, evidence-based care [12], and c) been exclusively or predominantly breastfed according to current recommendations. To achieve this goal, the INTERGROWTH-21 ${ }^{\text {st }}$ Neonatal Group developed, before the study commenced, a set of minimum recommendations for neonatal and postnatal care, based on current practice and recent publications (Table 1) [12-15]. Their feeding recommendations promoted exclusive breastfeeding by the time of hospital discharge and up to 6 months of age, consistent with the recommendations of WHO that have been adopted widely. Food source recommendations were, in order of preference, breastfeeding or expressed breast milk, donor human milk, and preterm formula. Guidelines for the initiation of milk feeding and progression volumes, and the use of nutritional supplements and fortifiers were provided $[12,15]$.

\section{Data collection}

Follow-up visits were scheduled $48-72 \mathrm{~h}$ after birth, every 2 weeks (i.e. 2 weeks \pm 2 days, $4 \pm 3,6 \pm 4$, and 8 weeks \pm 5 days from birth) during the first 8 weeks after birth, then every 4 weeks (i.e. 3 months \pm 9 days, $4 \pm 12,5 \pm 15,6 \pm 18,7 \pm 21$, and 8 months \pm 24 days after birth) until 8 months of postnatal age. Standardised questionnaires, used at each visit, recorded feeding practices and morbidity (available from www.intergrowth21.org.uk). Mothers were asked to recall the food 
Table 1 Evidence-based recommendations used in Preterm Postnatal Follow-up Study of the INTERGROWTH-21 $1^{\text {st }}$ Project

The overall goal of this guideline is to promote exclusive breastfeeding of the preterm infant by the time of hospital discharge.

What to feed, in order 1. Mother's own milk from the breast.

of preference $\quad 2$. Mother's expressed breast milk.

3. Donor human milk, if available.

4. Preterm formula.

How to feed

Orogastric feeding is preferred over a nasogastric tube, especially if the infant has increased work of breathing. Continuous, over-bolus feeds not recommended, no strong evidence for this. Infants should be encouraged to suck at the breast once sucking behavior is observed.

Feed volume

Start with approximately $80 \mathrm{ml} / \mathrm{kg}$ per day. Increase by $10-20 \mathrm{ml} / \mathrm{kg}$ per day to a maximum of approximately $160-180 \mathrm{ml} / \mathrm{kg}$ per day by the end of the first week of life.

Feed progression

Most infants $>32$ weeks' gestation will tolerate maintenance enteral feeds from the first day of life.

Infants $<32$ weeks' gestation should be introduced to small amounts of trophic feeds (10-24 ml/kg per day) on first day of life. Infants $>32$ weeks' gestation are likely to tolerate faster increases in volume.

Human milk supplementation

$$
\begin{aligned}
& \text { Vitamin D: } 400 \text { IU per day. } \\
& \text { Phosphorus \& calcium: } \\
& \text { some evidence for reducing metabolic } \\
& \text { bone disease in infants weighing <1,500 g.Iron: } \\
& 2 \mathrm{mg} / \mathrm{kg} \text { per day, start by } 8 \text { weeks of age. } \\
& \text { Multi-component fortifiers: Associated with } \\
& \text { short-term increases in weight gain, linear } \\
& \text { growth and head growth. No evidence for I } \\
& \text { ong-term benefits or adverse effects. }
\end{aligned}
$$

Duration of exclusive 6 months in low birth weight infants breastfeeding accompanied by iron supplementation.

types consumed, methods of feeding, use of vitamin and mineral supplements, and dietary intake of their infant in the 24-h period preceding the visit, using an Infant Food Recall (IFR) questionnaire. If their diet on that day was atypical (e.g. due to illness or travelling), mothers were asked to recall the last typical day. Anthropometric measurements (weight, length and head circumference) were taken as in WHO MGRS [16].

\section{Variable definitions and classifications}

Feeding practices were defined according to $\mathrm{WHO}$ indicators [17]. Exclusive breastfeeding refers to exclusive human breast milk with no other foods or liquids (including water), except oral rehydration solution and drops/syrups of vitamins, minerals or medicines. Predominantly breastfed refers to breast milk as the main source of nourishment but additional water or waterbased drinks, such as teas or infusions, fruit juice or ritual fluids, were offered. Partial breastfeeding refers to some breast milk as well as other foods or food-based fluids, such as formula milk or weaning foods. Formula and other liquids refer to predominantly received standard-, preterm-, high-energy-, soy-based-, hydrolysed-, or other special- formulas for preterm and low-birthweight infants, plus water, fruit or vegetable juice, tea or sweetened drinks.

Complementary foods included all semi-solid or solid foods besides milk or formula, except water, fruit or vegetable juice, tea and sweetened drinks and were categorised into seven groups: grains, roots and tubers; legumes and nuts; dairy products; flesh foods; eggs; vitamin A-rich fruits and vegetables; and other fruits and vegetables.

\section{Data analysis}

The mean and standard deviation (SD), or median and interquartile range (IQR), were used to describe continuous variables, and percentages for categorical variables. Where appropriate, the infants were categorised according to gestational age into early $\left(<34^{+0}\right.$ weeks' gestation) and late $\left(\geq 34^{+0}\right.$ to $<37^{+0}$ weeks' gestation) preterms.

\section{Ethical approval and consent}

The INTERGROWTH-21 $1^{\text {st }}$ Project was approved by the Oxfordshire Research Ethics Committee "C" (reference 08/H0606/139), the research ethics committees of the individual participating institutions, and the corresponding regional or national health authorities where the project was carried out. We obtained institutional consent to use routinely collected data and women gave oral consent.

\section{Results}

In total, 224 preterms were born in FGLS. Of these, 201 (99 boys and 102 girls) were alive, free of congenital malformations and enrolled in PPFS. The distribution of the 201 preterm newborns across sites was: Brazil $=18 \%$; China $=9 \%$; India $=15 \%$; Italy $=12 \%$; Kenya $=15 \%$; Oman $=15 \%$; UK $=11 \%$, and USA $=5 \%$. We documented feeding practices of these infants during their first 8 months after birth.

Most mothers (45.3\%) were aged 25-30 years and $72.6 \%$ were nulliparous; $30.4 \%$ had pregnancy-related and $19.9 \%$ non-pregnancy-related complications (Table 2). Of those with pregnancy-related complications, the most common were pregnancy-induced hypertension (8.5\%), vaginal bleeding $>27$ weeks' gestation (5.5\%), gestational diabetes $(5.0 \%)$, and pre-eclampsia $(3.0 \%$ ) (data not shown). At birth, the mean (SD) birthweight was 2,452 (519) g, length $45.6(2.7) \mathrm{cm}$, and head circumference 31.7 (1.8) $\mathrm{cm}$ (Table 2). The population was mostly composed of late preterm infants, as the median gestational age (GA) was 36 weeks (IQR 35.0 - 36.6).

Forty-seven percent of newborns were admitted to an intensive or special care unit after birth for respiratory support $(10.1 \%)$, surfactant treatment $(2.4 \%)$ or tube 
Table 2 Neonatal and maternal characteristics

\begin{tabular}{|c|c|c|}
\hline Variables & $N(N=201)$ & Value \\
\hline \multicolumn{3}{|l|}{ Maternal } \\
\hline \multicolumn{3}{|l|}{ Mother's age at delivery (\%) } \\
\hline $19-24$ & 38 & 18.9 \\
\hline $25-30$ & 91 & 45.3 \\
\hline $31-36$ & 72 & 35.8 \\
\hline \multicolumn{3}{|l|}{ Parity (\%) } \\
\hline 0 & 146 & 72.6 \\
\hline 1 & 41 & 20.4 \\
\hline 2 or more & 14 & 7.0 \\
\hline Pregnancy-related complications (\%) & 61 & 30.4 \\
\hline Non-pregnancy-related complications (\%) & 40 & 19.9 \\
\hline \multicolumn{3}{|l|}{ Neonatal } \\
\hline Girls (\%) & 102 & 50.7 \\
\hline Gestational age at birth (weeks) (median, IQR) & 201 & $36.0(35.0-36.6$ \\
\hline Early preterm, <34 weeks (\%) & 28 & 13.9 \\
\hline Late preterm, $\geq 34$ weeks and $<37$ weeks (\%) & 173 & 86.1 \\
\hline Birthweight $(g)^{\mathrm{a}}(\text { mean } \pm \mathrm{SD})^{\mathrm{a}}$ & 200 & $2452( \pm 519)$ \\
\hline Head circumference $(\mathrm{cm})^{\mathrm{a}}(\text { mean } \pm \mathrm{SD})^{\mathrm{a}}$ & 193 & $31.7( \pm 1.8)$ \\
\hline Length $(\mathrm{cm})^{\mathrm{a}}(\text { mean } \pm \mathrm{SD})^{\mathrm{a}}$ & 191 & $45.6( \pm 2.7)$ \\
\hline Apgar at 5 min $(\text { mean } \pm S D)^{a}$ & 200 & $9.2( \pm 0.9)$ \\
\hline Days until hospital discharge (median, IQR) & 201 & $4.0(2.0-8.0)$ \\
\hline Intensive/special care unit admission > 1 day (\%) & 82 & 40.7 \\
\hline Tube feeding or parenteral nutrition (\%) & 44 & 21.9 \\
\hline Hyperbilirubinemia (\%) & 46 & 22.9 \\
\hline Respiratory distress syndrome (\%) & 21 & 10.4 \\
\hline No oral feeds for $>24 \mathrm{~h}$ but $<7$ days (\%) & 24 & 11.9 \\
\hline Transient tachypnea of the newborn (\%) & 30 & 14.9 \\
\hline Hypoglycemia (\%) & 16 & 8.0 \\
\hline Apnea of prematurity (\%) & 14 & 7.0 \\
\hline Anaemia requiring transfusion (\%) & 2 & 1.0 \\
\hline Patent ductus arteriosus requiring pharmacological treatment (\%) & 4 & 2.0 \\
\hline Hypotension requiring inotropic treatment or steroids (\%) & 3 & 1.5 \\
\hline Bronchopulmonary dysplasia (\%) & 1 & 0.5 \\
\hline Other infection (\%) & 2 & 1.0 \\
\hline Meconium aspiration with respiratory distress (\%) & 1 & 0.5 \\
\hline Intraventricular hemorrhage grade I-II (\%) & 2 & 1.0 \\
\hline Retinopathy of prematurity (\%) & 1 & 0.5 \\
\hline Pneumonia/Bronchiolitis (\%) & 5 & 2.5 \\
\hline Stoppage of enteral feeding for $>3$ consecutive days (\%) & 2 & 1.0 \\
\hline
\end{tabular}

\footnotetext{
${ }^{\mathrm{a}}$ Missing data
}

${ }^{b}$ With normal growth and development

feeding/parenteral nutrition (34.3\%). The most common neonatal problems were hyperbilirubinemia (22.9 \%), respiratory distress (10.4\%), lack of oral feeding for $>24 \mathrm{~h}$ (11.9\%), and transient tachypnea (14.9\%). The median
(IQR) length of stay at special care units was 4.0 (2-8) days; three (1.5\%) preterms stayed in hospital for $\geq 60$ days (data not shown). As expected, most of the postnatal morbidities known to be related to severe 
prematurity (e.g. bronchopulmonary dysplasia, apnea of prematurity, retinopathy of prematurity) were observed in the very preterm infants (GA $<32$ weeks)

Within the first $72 \mathrm{~h}$ after birth, oral feeding was initiated in $94 \%$ of the infants. The percentage of infants who received any breastfeeding was $81.8 \%$ within $72 \mathrm{~h}$ of birth, $96.4 \%$ at 2 weeks of age, $93.5 \%$ at 8 weeks and $82 \%$ at 4 months (Fig. 1). Exclusive breastfeeding increased from $51.1 \%$ at $72 \mathrm{~h}$ after birth (Fig. 1) to $72.1 \%$ by hospital discharge, (late preterms $=75.7 \%$ and early preterms $=50.0 \%$, data not shown), with a further $16.9 \%$ partially breastfed at hospital discharge (late preterms $=15.0 \%$ and early preterms $=28.6 \%$, data not shown) (Fig. 2). The rate of exclusive breastfeeding continued to increase to $64.1 \%$ at 2 weeks, and was sustained until 3 months of postnatal age in $55.5 \%$ of the cohort (57.6 and $44.0 \%$ of late and early preterms, respectively; data not shown). At 4 weeks, $31.1 \%$ of infants were fed with breast milk and other milk/formula only, decreasing to $26.0 \%$ at 8 weeks, $31.7 \%$ at 3 months, $26.7 \%$ at 4 months and $10.3 \%$ at 6 months (Fig. 1). Exclusive breastfeeding gradually diminished between 5 and 8 months from 36.8 to $0.8 \%$ and 41.7 to $4.6 \%$, in late and early preterms, respectively (data not shown), while the consumption of semi-solid and solid foods increased from $32.3 \%$ at 5 months to $96.5 \%$ at 8 months (37.2 to $97.5 \%$, and 4.17 to $90.9 \%$, in late and early preterms, respectively) (Fig. 2). High rates of any breastfeeding continued with $73.7 \%$ still being breastfed at 6 months, and $70.4 \%$ (early preterms $=70.8 \%$ and late preterms $=68.2 \%$, data not shown) still receiving breast milk at 8 months of age (Fig. 2).

Grains, roots and tubers were consumed by $19.3 \%$ of preterms at 5 months, $51.1 \%$ at 6 months and $90.1 \%$ at
8 months of age (Fig. 3). By 5 months, vitamin A-rich fruits and vegetables and other fruits and vegetables were being consumed by 12.4 and $16.8 \%$, respectively. These two food groups were not offered as frequently as grains, roots and tubers, but were given to 61.3 and $72.5 \%$ of infants, respectively, by 8 months. Flesh foods, eggs, and legumes and nuts were offered to $36.8,7.9$, and $13.2 \%$ of infants at 7 months of age, respectively. By 8 months, flesh foods were given to $51.4 \%$ of preterms, while the consumption of eggs, and legumes and nuts remained low (14.1 and $19.7 \%$, respectively).

Iron supplements were routinely given to preterm infants at most sites in hospital and after discharge, except Oxford where, typically, only infants $<35$ weeks' gestation received supplements. Most sites provided Vitamin D supplements for infants after discharge but in the participating Nairobi hospitals, vitamin D was only given to selected preterms, and the practice was not standard in Oman. Vitamin $\mathrm{K}$ was given at birth to preterm babies at all sites, and most gave multivitamins during and after the hospital stay. Participating hospitals in Brazil gave multivitamins to selected preterms only; standard practice in China was Vitamin A supplementation in hospital and after discharge.

The patterns of longitudinal growth in this cohort for length were very similar to those of the WHO Child Growth Standards for term neonates [2] during the first months of life, but weight and head circumference differed from the WHO standards until 64 weeks' postmenstrual age [18].

\section{Discussion}

One of the aims of the INTERGROWTH-2 $1^{\text {st }}$ Project was to produce, for the first time, international standards for

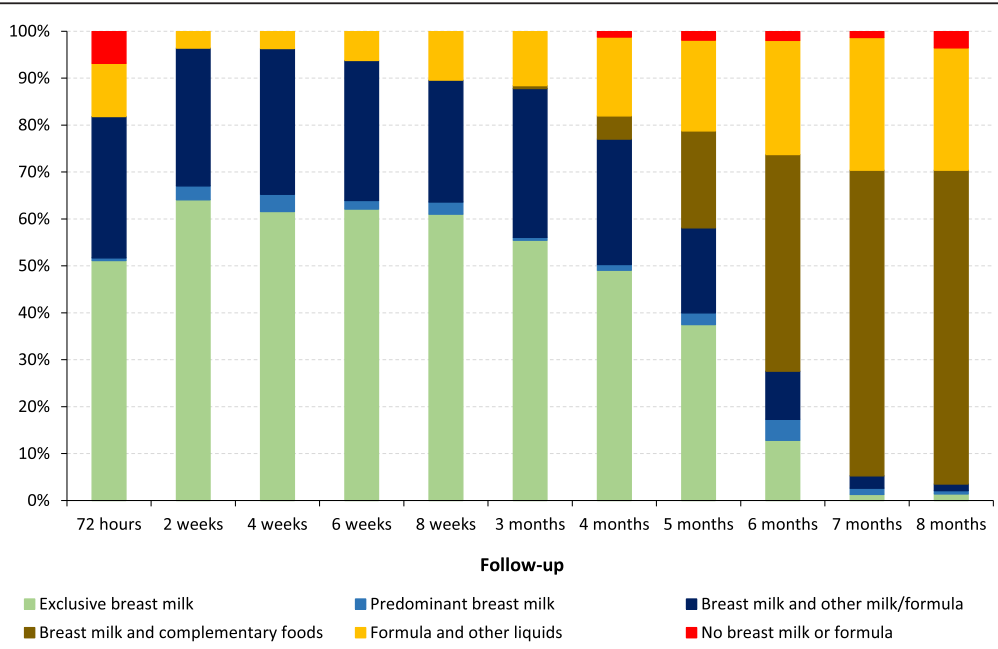

Fig. 1 Feeding practices of the preterm infants at each follow-up point. Parenteral nutrition or complementary foods refers to preterm infants receiving parenteral nutrition support during their first $72 \mathrm{~h}$, and infants receiving complementary foods only thereafter. Formula and other liquids refers to exclusive formula feeding during their first $72 \mathrm{~h}$ and formula with other liquids thereafter 


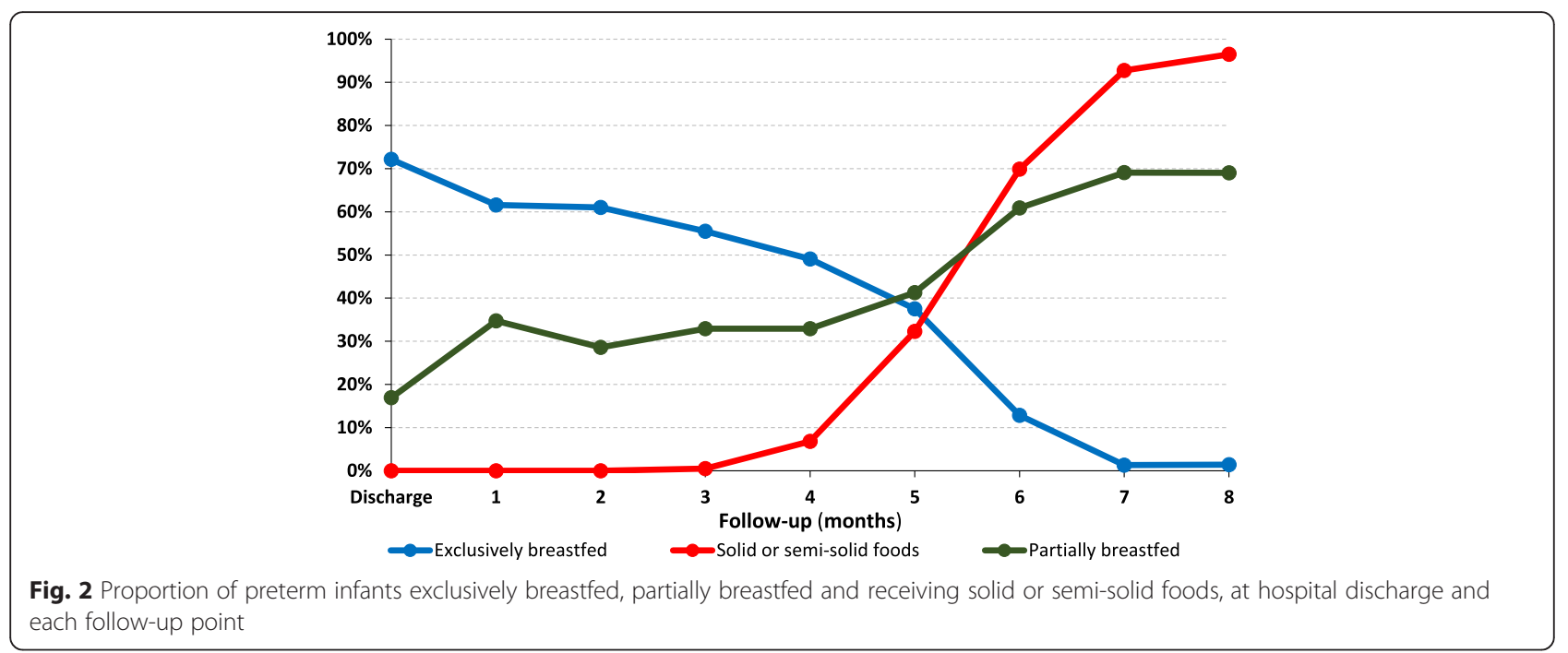

postnatal growth of preterm infants by gestational age, conforming to the prescriptive approach used in WHO MGRS. Healthy, well-nourished women with minimal constraints on fetal growth were studied prospectively, resulting in a relatively homogenous and 'healthy' population (according to gestational age) of preterm infants. To support the development of the standards, descriptive data were collected to document the degree to which feeding guidelines for adequate postnatal growth were followed.

The participating neonatal teams agreed to follow a set of recommended feeding practices based on the increasing evidence of the short- and long-term benefits of breastfeeding for preterm infants. This included guidelines on supplementation and the introduction of complementary foods.

In this cohort, nearly half the preterm infants were exclusively or predominantly breastfed until 4 months of age, and over two-thirds were still being breastfed at
8 months of age. The rates of exclusive or predominant breastfeeding were lower in this study than in WHO MGRS (MGRS - approximately 80 and $63 \%$; PPFS 65.3 and $56.5 \%$ at 4 weeks and 3 months, respectively) [19]. These results are not unexpected as breastfeeding rates are generally lower in preterm compared to term infants [9, 20-22], and establishing and maintaining breastfeeding in preterms requires considerable support. Factors that may impact upon the ability of preterms to breastfeed are clinical conditions related to prematurity, extended hospitalization, increased sleepiness and maternal illness. Other obstacles may include reduced muscular strength affecting latching, suckling and swallowing. The infant may also be discharged before lactation is fully established, so breastfeeding difficulties cannot be addressed by healthcare professionals. We emphasised these issues across the study sites, suggesting strategies to help mothers overcome these barriers.

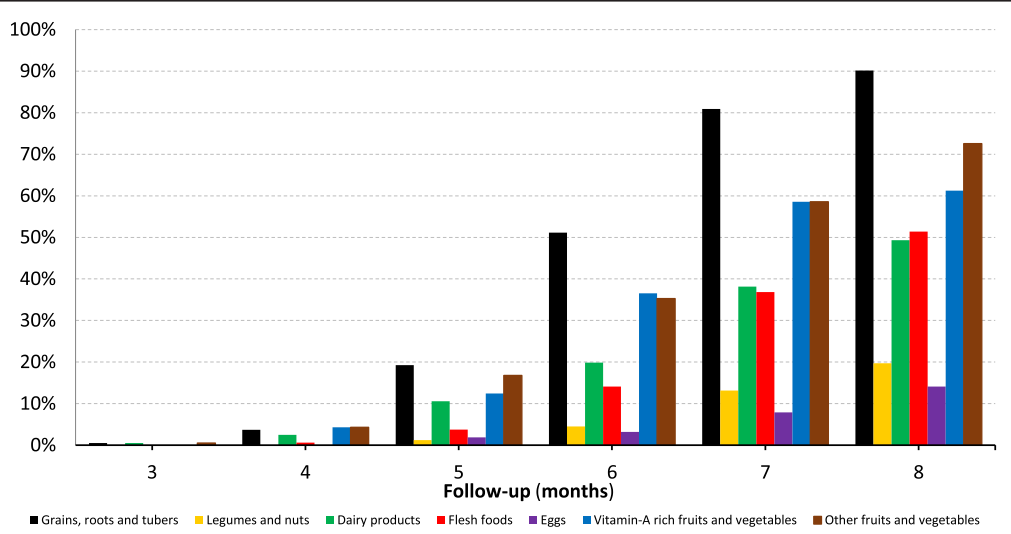

Fig. 3 Proportion of preterm infants consuming solid or semi-solid food groups, between 3 and 8 months of follow-up 
However, moving from the context of a research project to general practice will, realistically, be far more challenging.

It has been suggested that, wherever possible, preterm infants should be introduced to solid foods between 5 and 8 months of age to ensure that the appropriate transition period is not missed, and to encourage the development of feeding skills [23]. Healthy, late preterms, born $\geq 34$ weeks' gestation, may be introduced to solid foods using the same guidelines as term infants. The mean age at which complementary foods were introduced in PPFS was 6 months; not much later than reported for term infants (5.4 months) enrolled in a similar context, who achieved the recommended feeding criteria in WHO MGRS [24]. Of the complementary food groups, the grains, roots and tubers group was the most consistently offered, also similar to WHO MGRS.

Both the American Academy of Pediatrics and European Society of Paediatric Gastroenterology, Hepatology and Nutrition recommend preterm infants receive an iron supplement after hospital discharge $[25,26]$. Unlike exclusively breastfed term infants, who can usually maintain their iron levels until approximately 6 months of age, breastfed preterm infants should receive regular iron supplementation, 2$4 \mathrm{mg} / \mathrm{kg} /$ day, from hospital discharge until approximately 6 to 12 months of age [25-27]. Such recommendations were fully implemented at all INTERGROWTH-21 $1^{\text {st }}$ sites. There is some evidence to suggest that low birth weight infants and preterms $<32$ weeks' gestation may benefit from multivitamins and vitamin D supplementation [14]; nearly all our sites provided these supplements after hospital discharge, and vitamin $\mathrm{K}$ was given as standard at birth.

PPFS was designed to construct prescriptive standards from a population of preterm infants with the best potential for healthy growth and development, if supported by adequate nutrition and clinical care. Exclusive breastfeeding was established in most PPFS infants by hospital discharge and breastfeeding remained an important source of nutrition for these infants throughout the study. This was consistent with the main goal of the INTERGROWTH-21 $1^{\text {st }}$ Neonatal Group feeding guidelines [12], which were based on the most current evidence to promote optimal growth at the population level for uncomplicated preterm infants [14].

By 64 weeks' postmenstrual age, the $50^{\text {th }}$ centiles of our cohort's weights, lengths and head circumferences merged with those of the WHO Child Growth Standards [2], which indicates that preterm infants without severe postnatal complications, who are predominantly breastfed and receiving adequate postnatal care, progressively recuperate in early postnatal life [18].

\section{Conclusions}

There is little high-quality information available regarding the most appropriate patterns of postnatal growth for the optimal development and long-term health of preterm infants. Current evidence advocates breastfeeding with appropriate supplementation as the best way to support the growth and development of healthy preterm infants. When conducting postnatal growth research, it is essential to document feeding practices and the extent to which they adhered to the best available evidence.

Uncomplicated, preterm infants can follow evidencebased feeding recommendations centered on breastfeeding. When assessed against the WHO Child Growth Standards for 6 month old infants, adequate growth is achievable for babies born preterm with appropriate nutrition and clinical care.

\section{Appendix}

Members of the International Fetal and Newborn Growth Consortium for the $21^{\text {st }}$ Century (INTERGROWTH-21 $1^{\text {st }}$ ) and its Committees

\section{Scientific Advisory Committee}

M Katz (Chair from January 2011), MK Bhan, C Garza, S Zaidi, A Langer, PM Rothwell (from February 2011), Sir D Weatherall (Chair until December 2010).

\section{Steering Committee}

ZA Bhutta (Chair), J Villar (Principal Investigator), S Kennedy (Project Director), DG Altman, FC Barros, E Bertino, F Burton, M Carvalho, L Cheikh Ismail, WC Chumlea, MG Gravett, YA Jaffer, A Lambert, P Lumbiganon, JA Noble, RY Pang, AT Papageorghiou, M Purwar, J Rivera, C Victora.

\section{Executive Committee}

J Villar (Chair), DG Altman, ZA Bhutta, L Cheikh Ismail, S Kennedy, A Lambert, JA Noble, AT Papageorghiou.

\section{Project Coordinating Unit}

J Villar (Head), S Kennedy, L Cheikh Ismail, A Lambert, AT Papageorghiou, M Shorten, L Hoch (until May 2011), HE Knight (until August 2011), EO Ohuma (from September 2010), C Cosgrove (from July 2011), I Blakey (from March 2011).

\section{Data Analysis Group}

DG Altman (Head), EO Ohuma, J Villar.

\section{Data Management Group}

DG Altman (Head), F Roseman, N Kunnawar, SH Gu, JH Wang, MH Wu, M Domingues, P Gilli, L Juodvirsiene, L Hoch (until May 2011), N Musee (until June 2011), H AlJabri (until October 2010), S Waller (until June 2011), C Cosgrove (from July 2011), D Muninzwa (from October 
2011), EO Ohuma (from September 2010), D Yellappan (from November 2010), A Carter (from July 2011), D Reade (from June 2012), R Miller (from June 2012).

\section{Ultrasound Group}

AT Papageorghiou (Head), L Salomon (Senior external advisor), A Leston, A Mitidieri, F Al-Aamri, W Paulsene, J Sande, WKS Al-Zadjali, C Batiuk, S Bornemeier, M Carvalho, M Dighe, P Gaglioti, N Jacinta, S Jaiswal, JA Noble, K Oas, M Oberto, E Olearo, MG Owende, J Shah, S Sohoni, T Todros, M Venkataraman, S Vinayak, L Wang, D Wilson, QQ Wu, S Zaidi, Y Zhang, P Chamberlain (until September 2012), D Danelon (until July 2010), I Sarris (until June 2010), J Dhami (until July 2011), C Ioannou (until February 2012), CL Knight (from October 2010), R Napolitano (from July 2011), S Wanyonyi (from May 2012), C Pace (from January 2011), V Mkrtychyan (from June 2012).

\section{Anthropometry Group}

L Cheikh Ismail (Head), WC Chumlea (Senior external advisor), F Al-Habsi, ZA Bhutta, A Carter, M Alija, JM Jimenez-Bustos, J Kizidio, F Puglia, N Kunnawar, H Liu, S Lloyd, D Mota, R Ochieng, C Rossi, M Sanchez Luna, YJ Shen, HE Knight (until August 2011), DA Rocco (from June 2012), IO Frederick (from June 2012).

\section{Neonatal Group}

ZA Bhutta (Head), E Albernaz, M Batra, BA Bhat, E Bertino, P Di Nicola, F Giuliani, I Rovelli, K McCormick, R Ochieng, RY Pang, V Paul, V Rajan, A Wilkinson, A Varalda (from September 2012).

\section{Environmental Health Group}

B Eskenazi (Head), LA Corra, H Dolk, J Golding, A Matijasevich, T de Wet, JJ Zhang, A Bradman, D Finkton, O Burnham, F Farhi.

\section{Participating countries and local investigators}

Brazil: FC Barros (Principal Investigator), M Domingues, S Fonseca, A Leston, A Mitidieri, D Mota, IK Sclowitz, MF da Silveira.

China: RY Pang (Principal Investigator), YP He, Y Pan, YJ Shen, MH Wu, QQ Wu, JH Wang, Y Yuan, Y Zhang.

India: M Purwar (Principal Investigator), A Choudhary, S Choudhary, S Deshmukh, D Dongaonkar, M Ketkar, V Khedikar, N Kunnawar, C Mahorkar, I Mulik, K Saboo, C Shembekar, A Singh, V Taori, K Tayade, A Somani.

Italy: E Bertino (Principal Investigator), P Di Nicola, M Frigerio, G Gilli, P Gilli, M Giolito, F Giuliani, M Oberto, L Occhi, C Rossi, I Rovelli, F Signorile, T Todros.

Kenya: W Stones and M Carvalho (Co- Principal Investigators), J Kizidio, R Ochieng, J Shah, S Vinayak, N
Musee (until June 2011), C Kisiang'ani (until July 2011), D Muninzwa (from August 2011).

Oman: YA Jaffer (Principal Investigator), J Al-Abri, J AlAbduwani, FM Al-Habsi, H Al-Lawatiya, B Al-Rashidiya, WKS Al-Zadjali, FR Juangco, $\mathrm{M}$ Venkataraman, $\mathrm{H}$ AlJabri (until October 2010), D Yellappan (from November 2010).

UK: S Kennedy (Principal Investigator), L Cheikh Ismail, AT Papageorghiou, F Roseman, A Lambert, EO Ohuma, S Lloyd, R Napolitano (from July 2011), C Ioannou (until February 2012), I Sarris (until June 2010).

USA: MG Gravett (Principal Investigator), C Batiuk, M Batra, S Bornemeier, M Dighe, K Oas, W Paulsene, D Wilson, IO Frederick, HF Andersen, SE Abbott, AA Carter, $\mathrm{H}$ Algren, DA Rocco, TK Sorensen, D Enquobahrie, S Waller (until June 2011).

\section{Competing interests}

The authors declare that they have no competing interests.

\section{Authors' contributions}

All authors read and approved the final manuscript.

\section{Acknowledgements}

This project was supported by a generous grant from the Bill \& Melinda Gates Foundation to the University of Oxford, for which we are very grateful. We would also like to thank the Health Authorities in Pelotas, Brazil; Beijing, China; Nagpur, India; Turin, Italy; Nairobi, Kenya; Muscat, Oman; Oxford, UK and Seattle, USA, who facilitated the project by allowing participation of these study sites as collaborating centres. We are extremely grateful to Philips Medical Systems who provided the ultrasound equipment and technical assistance throughout the project. We also thank MedSciNet U.K. Ltd for setting up the INTERGROWTH-21 $1^{\text {st }}$ web-site and for the development, maintenance and support of the on-line data management system. MMS gratefully acknowledges the financial support of the National Counsel of Technological and Scientific Development, Brazil.

We thank the parents and infants who participated in the studies and the more than 200 members of the research teams who made the implementation of this project possible. The participating hospitals included: Brazil, Pelotas (Hospital Miguel Piltcher, Hospital São Francisco de Paula, Santa Casa de Misericórdia de Pelotas, and Hospital Escola da Universidade Federal de Pelotas); China, Beijing (Beijing Obstetrics \& Gynecology Hospital, Shunyi Maternal \& Child Health Centre, and Shunyi General Hospital); India, Nagpur (Ketkar Hospital, Avanti Institute of Cardiology Private Limited, Avantika Hospital, Gurukrupa Maternity Hospital, Mulik Hospital \& Research Centre, Nandlok Hospital, Om Women's Hospital, Renuka Hospital \&

Maternity Home, Saboo Hospital, Brajmonhan Taori Memorial Hospital, and Somani Nursing Home); Kenya, Nairobi (Aga Khan University Hospital, MP Shah Hospital and Avenue Hospital); Italy, Turin (Ospedale Infantile Regina Margherita Sant' Anna and Azienda Ospedaliera Ordine Mauriziano); Oman, Muscat (Khoula Hospital, Royal Hospital, Wattayah Obstetrics \& Gynaecology Poly Clinic, Wattayah Health Centre, Ruwi Health Centre, Al-Ghoubra Health Centre and Al-Khuwair Health Centre); UK, Oxford (John Radcliffe Hospital) and USA, Seattle (University of Washington Hospital, Swedish Hospital, and Providence Everett Hospital).

Full acknowledgement of all those who contributed to the development of the INTERGROWTH-21 $1^{\text {st }}$ Project protocol appears at

www.intergrowth21.org.uk.

Financial disclosure statement

The authors have indicated that they have no financial relationships relevant to this article to disclose.

\section{Author details}

${ }^{1}$ Nuffield Department of Obstetrics \& Gynaecology and Oxford Maternal \& Perinatal Health Institute, Green Templeton College, University of Oxford, 
Oxford, UK. ${ }^{2}$ Neonatologia Universitaria, Dipartimento di Scienze della Sanità Pubblicae Pediatriche, Università degli Studi di Torino, Torino, Italy. ${ }^{3}$ Royal Hospital, Muscat, Sultanate of Oman. ${ }^{4}$ Faculty of Health Sciences, Aga Khan University, Nairobi, Kenya. ${ }^{5}$ Centre for Statistics in Medicine, Botnar Research Centre, University of Oxford, Oxford, UK. ${ }^{6}$ Department of Engineering Science, University of Oxford, Oxford, UK. ${ }^{7}$ Global Alliance to Prevent Prematurity and Stillbirth (GAPPS), Seattle Children's, Seattle, WA, USA. ${ }^{8}$ Nagpur INTERGROWTH-21st Research Centre, Ketkar Hospital, Nagpur, India. ${ }^{9}$ Shunyi Maternal \& Child Health Hospital, Beijing, China. ${ }^{10}$ Departamento Materno-Infantil, Universidade Federal de Pelotas, Pelotas, RS, Brazil. ${ }^{11}$ Center of Excellence in Women \& Child Health, Aga Khan University, Karachi, Pakistan and Center for Global Child Health, Hospital for Sick Children, Toronto, Canada. ${ }^{12}$ Nuffield Department of Obstetrics \& Gynaecology, Level 3 Women's Centre, John Radcliffe Hospital, Oxford OX3 9DU, UK.

Received: 23 June 2015 Accepted: 21 January 2016

Published online: 05 February 2016

\section{References}

1. Villar J, Altman DG, Purwar M, Noble JA, Knight HE, Ruyan P, et al. International Fetal and Newborn Growth Consortium for the $21^{\text {st }}$ Century: The objectives, design and implementation of the INTERGROWTH-21 ${ }^{\text {st }}$ Project. BJOG. 2013;120 Suppl 2:9-26.

2. de Onis M, Garza C, Onyango AW, Martorell R. WHO Child Growth Standards. Acta Paediatr Suppl. 2006;450:1-101.

3. de Onis M, Garza C, Victora CG, Bhan MK, Norum KR. The WHO Multicentre Growth Reference Study: planning, study design, and methodology. Food Nutr Bull. 2004;25(1 Suppl):S15-26.

4. Villar J, Knight HE, de Onis M, Bertino E, Gilli G, Papageorghiou AT, et al. International Fetal and Newborn Growth Consortium (INTERGROWTH-21 ${ }^{\text {st }}$ ) Conceptual issues related to the construction of prescriptive standards for the evaluation of postnatal growth of preterm infants. Arch Dis Child. 2010; 95(12):1034-8

5. Eskenazi B, Bradman A, Finkton D, Purwar M, Noble JA, Pang $R$, et al. International Fetal and Newborn Growth Consortium for the $21^{\text {st }}$ Century. A rapid questionnaire assessment of environmental exposures to pregnant women in the INTERGROWTH-21 ${ }^{\text {st }}$ Project. BJOG. 2013;120 Suppl 2:129-38

6. Santos IS, Matijasevich A, Domingues MR, Barros AJ, Victora CG, Barros FC. Late preterm birth is a risk factor for growth faltering in early childhood: a cohort study. BMC Pediatr. 2009;9:71.

7. Tudehope D, Fewtrell M, Kashyap S, Udaeta E. Nutritional needs of the micropreterm infant. J Pediatr. 2013;162(3 Suppl):S72-80.

8. Ward Platt M, Deshpande S. Metabolic adaptation at birth. Semin Feta Neonatal Med. 2005;10(4):341-50.

9. Lapillonne A, O'Connor DL, Wang D, Rigo J. Nutritional recommendations for the late-preterm infant and the preterm infant after hospital discharge. J Pediatr. 2013;162(3 Suppl):S90-100.

10. Clark RH, Thomas $P$, Peabody J. Extrauterine growth restriction remains a serious problem in prematurely born neonates. Pediatrics. 2003;111(5 Pt 1): 986-90.

11. Underwood MA. Human milk for the premature infant. Pediatr Clin North Am. 2013;60(1):189-207.

12. Bhutta Z, Giuliani F, Haroon A, Knight HE, Albernaz E, Batra M, et al. International Fetal and Newborn Growth Consortium for the $21^{\text {st }}$ Century. Standardisation of neonatal clinical practice. BJOG. 2013;120 Suppl 2:56-63.

13. WHO, UNICEF. Global Strategy on Infant and Young Child Feeding. Geneva: WHO Press; 2003.

14. Edmond K, Bahl R. World Health Organization Technical Review: Optimal feeding of low-birth-weight infants. Geneva: WHO Press; 2006.

15. INTERGROWTH-21 $21^{\text {st }}$ Neonatal Care Group. Basic Neonatal Care Manual. 2010. http://www.medscinet.net/Intergrowth/patientinfodocs/ Neonatal\%20Manual\%20Final.pdf. Accessed 12 June 2015

16. Cheikh Ismail L, Knight H, Ohuma E, Hoch L, Chumlea WC. International Fetal and Newborn Growth Consortium for the $21^{\text {st }}$ Century. Anthropometric standardisation and quality control protocols for the construction of new, international, fetal and newborn growth standards: the INTERGROWTH-21 $1^{\text {st }}$ Project. BJOG. 2013;120 Suppl 2:48-55.

17. WHO. Indicators for assessing infant and young child feeding practices Part I: Definitions. Geneva: WHO Press; 2008.
18. Villar J, Giuliani F, Bhutta ZA, Bertino E, Ohuma EO, Cheikh Ismail L, et al Postnatal growth standards for preterm infants: the Preterm Postnatal Follow-up Study of the INTERGROWTH-21 ${ }^{\text {st }}$ Project. Lancet Global Health. 2015:3:e681-91.

19. WHO Multicentre Growth Reference Study Group. Breastfeeding in the WHO Multicentre Growth Reference Study. Acta Paediatr Suppl. 2006;450:16-26.

20. Meier P, Patel AL, Wright K, Engstrom JL. Management of breastfeeding during and after the maternity hospitalization for late preterm infants. Clin Perinatol. 2013;40(4):689-705.

21. Nagulesapillai T, McDonald SW, Fenton TR, Mercader HF, Tough SC. Breastfeeding difficulties and exclusivity among late preterm and term infants: results from the all our babies study. Can J Public Health. 2013; 104(4):e351-6.

22. Liu P, Qiao L, Xu F, Zhang M, Wang Y, Binns CW. Factors associated with breastfeeding duration: a 30-month cohort study in northwest China. J Hum Lact. 2013;29(2):253-9.

23. King CL. An evidence based guide to weaning preterm infants. Paediatr Child Health. 2009;19(9):405-14.

24. WHO Multicentre Growth Reference Study Group. Complementary feeding in the WHO Multicentre Growth Reference Study. Acta Paediatr Suppl. 2006; 450:27-37.

25. Agostoni C, Buonocore G, Carnielli VP, De Curtis M, Darmaun D, Decsi T, et al. ESPGHAN Committee on Nutrition. Enteral nutrient supply for preterm infants; commentary from the European Society of Paediatric Gastroenterology, Hepatology and Nutrition Committee on Nutrition. J Pediatr Gastroenterol Nutr. 2010:50(1):85-91.

26. Baker RD, Greer FR. Diagnosis and prevention of iron deficiency and irondeficiency anemia in infants and young children ( $0-3$ years of age). Pediatrics. 2010;126(5):1040-50.

27. Tsang RC, Koletzko B, Zlotkin S. Nutrition of the Preterm Infant: Scientific Basis and Practical Guidelines. Cincinatti, OH: Digital Educational Publishing Inc; 2005

\section{Submit your next manuscript to BioMed Central and we will help you at every step:}

- We accept pre-submission inquiries

- Our selector tool helps you to find the most relevant journal

- We provide round the clock customer support

- Convenient online submission

- Thorough peer review

- Inclusion in PubMed and all major indexing services

- Maximum visibility for your research

Submit your manuscript at www.biomedcentral.com/submit

) Biomed Central 